\title{
Informationsgehalt von Credit Ratings
}


Steffen Hundt

\section{Informationsgehalt von Credit Ratings}

Eine empirische Analyse europäischer Aktien- und Anleihemärkte

Mit einem Geleitwort von Prof. Dr. Andreas Horsch

Dinger Gabler 
Steffen Hundt

Freiberg, Deutschland

Dissertation Technische Universität Bergakademie Freiberg, 2015

ISBN 978-3-658-11309-4

ISBN 978-3-658-11310-0 (eBook)

DOI 10.1007/978-3-658-11310-0

Die Deutsche Nationalbibliothek verzeichnet diese Publikation in der Deutschen Nationalbibliografie; detaillierte bibliografische Daten sind im Internet über http://dnb.d-nb.de abrufbar.

Springer Gabler

(C) Springer Fachmedien Wiesbaden 2015

Das Werk einschließlich aller seiner Teile ist urheberrechtlich geschützt. Jede Verwertung, die nicht ausdrücklich vom Urheberrechtsgesetz zugelassen ist, bedarf der vorherigen Zustimmung des Verlags. Das gilt insbesondere für Vervielfältigungen, Bearbeitungen, Übersetzungen, Mikroverfilmungen und die Einspeicherung und Verarbeitung in elektronischen Systemen.

Die Wiedergabe von Gebrauchsnamen, Handelsnamen, Warenbezeichnungen usw. in diesem Werk berechtigt auch ohne besondere Kennzeichnung nicht zu der Annahme, dass solche Namen im Sinne der Warenzeichen- und Markenschutz-Gesetzgebung als frei zu betrachten wären und daher von jedermann benutzt werden dürften.

Der Verlag, die Autoren und die Herausgeber gehen davon aus, dass die Angaben und Informationen in diesem Werk zum Zeitpunkt der Veröffentlichung vollständig und korrekt sind. Weder der Verlag noch die Autoren oder die Herausgeber übernehmen, ausdrücklich oder implizit, Gewähr für den Inhalt des Werkes, etwaige Fehler oder Äußerungen.

Gedruckt auf säurefreiem und chlorfrei gebleichtem Papier

Springer Fachmedien Wiesbaden ist Teil der Fachverlagsgruppe Springer Science+Business Media (www.springer.com) 


\section{Geleitwort}

Credit Ratings sollen den Parteien von Kreditverträgen helfen, die für Kredite arttypischen Informationsasymmetrien zu handhaben. Das Bedürfnis nach solchen Kapitaldienstabschätzungen hat im Laufe der Zeit stetig zugenommen und hat den hierauf spezialisierten Credit Rating Companies seit Mitte des 19. Jahrhunderts einen ebenso stetigen Bedeutungszuwachs beschert. Zusätzlich aufgewertet wurden diese Akteure durch die regulatorische Indienstnahme von Ratings seit den 1970er Jahren. Diese rating-basierte Regulierung (von Finanzintermediären) trieben verschiedene Regelsetzer voran, obgleich die Credit Rating Companies regelmäßig im Zuge von Unternehmens- bzw. Finanzmarktkrisen zu den Akteuren gezählt wurden. Unabhängig davon, dass der Umgang gerade politischer Entscheidungsträger mit Credit Ratings nicht frei von Widersprüchen ist, ist er von der Auffassung geprägt, dass diesen Bonitätsurteilen ein Informationsgehalt zukommt.

Nicht nur vor dem Hintergrund der jüngsten Krisenprozesse auf den Finanzmärkten stellt sich daher die Frage, inwieweit (Veränderungen von) Credit Ratings die Wissensbestände von Marktteilnehmern tatsächlich soweit verändern, dass diese sich zu Handlungen veranlasst sehen. Derart rating-induzierten Käufen und Verkäufen und der daraus resultierenden Preisentwicklung der betroffenen Wertpapiere geht Steffen Hundt in seiner Dissertationsschrift nach. Seine Arbeit zeichnet sich damit gleichermaßen durch Aktualität wie durch ökonomische Relevanz aus. Das solide Fundament seiner empirischen Analysen bildet die Theorie effizienter Kapitalmärkte sowie der Finanzintermediation einerseits sowie eine lobenswert umfassende und präzise Rezeption des bisherigen Forschungsgeschehens andererseits. Auf dieser Basis entwickelt Steffen Hundt einen Hypothesenapparat, den er mit Hilfe der etablierten Methodik des Ereignisstudienansatzes empirischen Tests unterzieht. Im Vergleich zur Majorität bisheriger Arbeiten heben sich diese bereits durch ihren europäischen Fokus positiv ab. Hinzu kommen die Differenzierung von Vor- und Nachkrisenphasen sowie insbesondere die vergleichende Analyse von Emissionen verschiedener Wertpapierkategorien ein und desselben Emittenten. Auf diese Weise werden zum einen generelle Unterschiede und Gemeinsamkeiten im Verhalten der Investoren bzw. Marktpreise verschiedener Wertpapierkategorien herausgearbeitet. Zum anderen wird speziell für die bisher von der Forschung vernachlässigten Wandelanleihen geklärt, ob sich diese hybriden Instrumente eher anleihe- oder eher aktienähnliche Reaktionsmuster aufweisen. Es resultieren Erkenntnisse, die nicht nur für die Erklärung bisheriger, sondern auch für die Prognose zukünftiger Preisentwicklungen im Zuge von Rating-Änderungen von erheblichem Interesse sind. Die Erkenntnisse erscheinen umso gewichtiger, als sich im Zuge der krisengetriebenen Re-Regulierung von Financial Institutions ein erheblicher Bedeutungszuwachs für besondere Formen von Wandelanleihen ankündigt.

Gerade die Krisenprozesse auf den Finanzmärkten der letzten Jahre haben unterstrichen, dass eine seriöse Neubewertung der Relevanz von Credit Ratings dringend geboten ist. Steffen Hundt erweitert den dafür heranziehbaren Wissensstand zum Informationsgehalt von Credit 
Ratings in mehrerlei und bedeutsamer Hinsicht. Seiner Arbeit, die von der wirtschaftswissenschaftlichen Fakultät der Technischen Universität Bergakademie Freiberg im April 2015 als Dissertation angenommen wurde, wünsche ich daher eine entsprechend weite Verbreitung sowie geneigte Rezeption in Wissenschaft und Praxis.

Andreas Horsch 


\section{Vorwort}

Nicht zuletzt die jüngst gescheiterten Versuche zur Etablierung eines europäischen RatingUnternehmens haben die dominante Rolle der drei führenden amerikanischen Rating Agencies auf europäischen Kapitalmärkten verdeutlicht. Als Einschätzung des Bonitätsrisikos sind sie integraler Bestandteil von Tauschbeziehungen zwischen Kapitalgeber und -nehmer. Hierbei liegt der Fokus nicht auf der Bonitätseinschätzung zu einem bestimmten Zeitpunkt, sondern vielmehr auf der Bonitätsentwicklung über einem bestimmten Zeitraum. Eine veränderte Bonität des Rating-Objektes müsste sich vor diesem Hintergrund auch in einer Veränderung des Credit Ratings widerspiegeln. Aus Sicht der Kapitalnehmer wird der Stellenwert von Credit Ratings primär durch ihren Einfluss auf die Finanzierungskosten determiniert. Hingegen steht für Kapitalgeber der Abbau von Unsicherheiten durch Nutzung dieser externen Bonitätsurteile im Vordergrund. Als Information über ein verändertes Kreditrisiko eines bestimmten Rating-Objektes ist somit zu erwarten, dass sich die Bedeutung von Rating-Änderungen auf Wertpapiermärkten aus Sicht der dort agierenden Wertpapierinvestoren in Kauf- bzw. Verkaufshandlungen und somit in veränderten Wertpapierpreisen bzw. -renditen manifestiert. Diese Kausalität ist insbesondere für europäische Wertpapiermärkte zu überprüfen, da den drei führenden amerikanischen Rating-Unternehmen pars pro toto fehlende Kenntnis der Marktstrukturen sowie -prozesse europäischer Kapitalmärkte vorgeworfen wird. Die sich hieraus ergebende Frage nach dem Informationsgehalt von Rating-Änderungen ist bereits Gegenstand bisheriger Studien gewesen, wenngleich bisher nahezu ausschließlich Kurs- bzw. Renditeeffekte von Aktien und Unternehmensanleihen amerikanischer Emittenten untersucht wurden. Fokus dieser Arbeit ist neben Aktien und Unternehmensanleihen deshalb die Wandelanleihe als hybrides Wertpapier, das im Rahmen der Finanzierung europäischer Unternehmen zunehmend an Bedeutung gewinnt.

Im Rahmen einer modifizierten Ereignisstudie erfolgt die Analyse des Renditeeffektes von Wandelanleihen infolge angekündigter Rating-Änderungen. Die Resultate der Untersuchung zeigen, dass angekündigte Rating-Änderungen zu signifikanten Renditeeffekten auf europäischen Wandelanleihemärkten führen. Ferner wird in diesem Zusammenhang deutlich, dass die Richtung des Renditeeffektes maßgeblich von der Richtung der Rating-Änderung abhängt. Schließlich zeigt der Vergleich von Wandelanleihen mit Aktien und Unternehmensanleihen des gleichen Emittenten, dass nur vereinzelt Reaktionsunterschiede in Abhängigkeit der Wertpapierkategorie festzustellen sind. Diese Ergebnisse fungieren als Ausgangspunkt einer anschließenden ökonomischen Erläuterung, deren Ziel insbesondere darin besteht, wertpapierspezifische Ursachen für die jeweiligen Renditereaktionen zu identifizieren.

Entstanden ist diese Arbeit während meiner Zeit als wissenschaftlicher Mitarbeiter am Lehrstuhl für Allgemeine Betriebswirtschaftslehre mit dem Schwerpunkt Investition und Finanzierung an der TU Bergakademie Freiberg. Folglich gilt mein ganz besonderer Dank meinem hochgeschätzten akademischen Lehrer und Doktorvater Prof. Dr. Andreas Horsch. Seinen profunden Kenntnissen im Bereich der Kreditrisikoanalyse sowie seinem Gespür für Aktuali- 
tät habe ich es zu verdanken, dass diese Arbeit zu einer Zeit entstanden ist, in der die Debatte um die Notwendigkeit eines europäischen Rating-Unternehmens und der damit einhergehenden Kritik an der Rolle der drei führenden Rating Agencies auf europäischen Kapitalmärkten ihren vorläufigen Höhepunkt fand. Darüber hinaus danke ich Ihm für seine Herzlichkeit sowie seine offene und respektvolle Art, durch die sich vom ersten Tage der Zusammenarbeit eine Atmosphäre des Vertrauens entwickeln konnte. Ferner gilt meine Wertschätzung und Dankbarkeit Frau Prof. Dr. Silvia Rogler, deren Betreuung im Rahmen der Zweitbegutachtung dieser Arbeit eine ideale Ergänzung darstellte.

Meine Dankbarkeit gilt an dieser Stelle auch allen Freunden und Kollegen, die zum Gelingen dieser Arbeit beigetragen haben. Allen voran möchte ich meinen wissenschaftlichen Kollegen und lieben Freund Dipl.-Math. Björn Sprungk für die Unterstützung bei der Konzeption der statistischen Modellierung dieser empirischen Studie danken. Aufbauend hierauf freue ich mich sehr, dass wir unsere Zusammenarbeit im Laufe der Zeit durch gemeinsame Publikationsprojekte intensivieren konnten, was dieser Arbeit aus meiner Sicht einen besonderen Charme verleiht.

Einen unschätzbaren Beitrag hat schließlich meine Familie geleistet, deren Geduld und Ausdauer mir stets den notwendigen Rückhalt gegeben haben. Meine Lebensgefährtin Beatrix, meine Eltern Petra und Jochen, meine Oma Ursel sowie meine Schwester Susanne ließen mich durch Ihre Liebe und Ihre Unerschütterlichkeit auch und insbesondere in den schweren Phasen meiner Promotionszeit stets von Neuem Kraft schöpfen. Mein tiefster Dank gilt meiner Lebensgefährtin und besten Freundin Beatrix, die so vieles aushalten musste und mir doch so unendlich viel gegeben hat. Insbesondere die Tatsache, dass der Beginn meiner Promotion fast zeitgleich einherging mit dem Beginn unserer Beziehung, hat uns als Paar in einer frühen Phase einer ungewöhnlich großen Prüfung unterzogen, deren erfolgreiches Bestehen mich rückblickend ehrfürchtig erkennen lässt, dass ich in der Promotionszeit wahrlich die Liebe meines Lebens gefunden habe. In diesem Zusammenhang stellt die Geburt unserer Tochter Friederike Wilhelmine in der finalen Phase dieser Arbeit den krönenden Abschluss dar, den ich in Worte zu fassen nicht vermag. In ewiger Verbundenheit zu meiner Heimat Aken / Elbe widme ich diese Arbeit daher meiner Familie. Ich danke Euch allen von ganzem Herzen.

Steffen Hundt 


\section{Inhaltsverzeichnis}

Geleitwort..................................................................................................................... V

Vorwort ......................................................................................................................

Inhaltsverzeichnis........................................................................................................... IX

Abbildungsverzeichnis ................................................................................................... XIII

Abkürzungsverzeichnis.............................................................................................XVII

1. Einleitung ………........................................................................................................

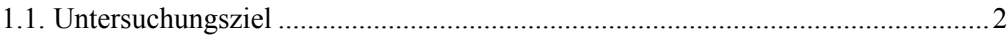

1.2. Gang der Untersuchung .............................................................................. 5

2. Informationen und Kapitalmärkte .................................................................................

2.1. Der Informationsbegriff...................................................................................

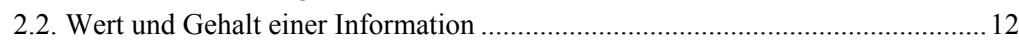

2.3. Der informationseffiziente Kapitalmarkt …………………………………...... 14

3. Grundlagen von Credit Ratings .....................................................................................20

3.1. Bonitätsrisiken als Ausgangspunkt von Credit Ratings ......................................2

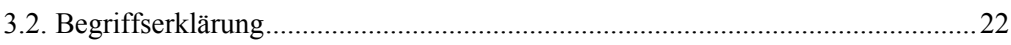

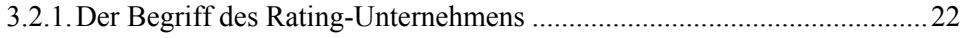

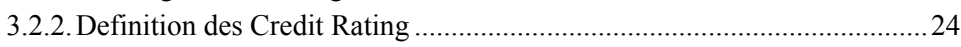

3.2.3. Definition der Rating-Änderung ................................................................... 30

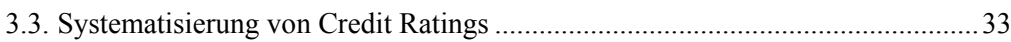

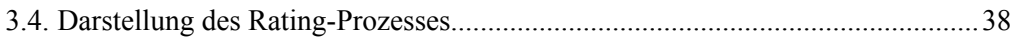

3.5. Struktur des europäischen Marktes für Credit Ratings......................................... 45

3.6. Kritische Analyse von Rating-Unternehmen ....................................................50

4. Existenzbegründung von Credit Ratings und verbriefter Finanzierung ..............54

4.1. Theoretische Begründung von Rating-Unternehmen .............................................54

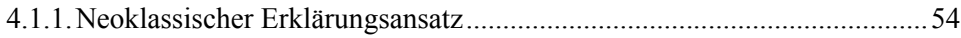

4.1.2. Neoinstitutionenökonomische Erklärungsansätze ......................................54

4.1.2.1. Intermediation durch Rating-Unternehmen ................................56

4.1.2.2. Transaktionskostenbasierter Erklärungsansatz ............................61

4.1.2.3. Agency-basierter Erklärungsansatz.................................................. 64

4.2. Theoretische Begründung der verbrieften Finanzierung........................................69

4.2.1. Verbriefte Finanzierung auf vollkommenen Kapitalmärkten ......................69 69

4.2.2. Verbriefte Finanzierung auf unvollkommenen Kapitalmärkten ..................71

5. Wirkung von angekündigten Rating-Änderungen auf Wertpapierkurse.............79

5.1. Bonitätsrisiko und die Bewertung von Wertpapieren ..........................................79 
5.1.1.Einfluss des Bonitätsrisikos auf den Aktienkurs..................................... 79

5.1.2. Einfluss des Bonitätsrisikos auf den Kurs von Unternehmensanleihen..... 80

5.1.3. Einfluss des Bonitätsrisikos auf den Kurs von Wandelanleihen................. 82

5.2. Determinanten des Informationsgehalts von Rating-Änderungen ...................... 85

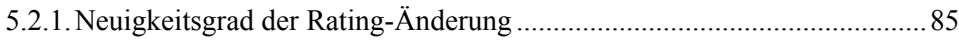

5.2.2. Interessenkonflikt zwischen Aktien- und Anleiheinvestoren .................... 90

5.2.3. Branchenzugehörigkeit des Wertpapieremittenten ................................... 95

5.2.4. Phasen innerhalb des Konjunkturzyklus ............................................... 102

5.2.5. Wertpapierliquidität als Maß der Informationsverarbeitung in

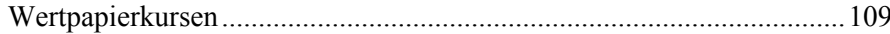

5.2.6. Sonstige Einflussfaktoren ................................................................... 116

5.3. Einfluss unabhängiger Variablen auf Wertpapierkurse im Rahmen von Rating-

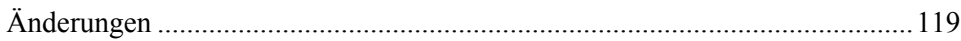

5.3.1. Einfluss unabhängiger Variablen auf Aktienkurse ............................... 120

5.3.2. Einfluss unabhängiger Variablen auf Unternehmensanleihekurse .......... 124

5.3.3. Einfluss unabhängiger Variablen auf Wandelanleihekurse ..................... 128

5.4. Erwartete Wirkung identifizierter Einflussfaktoren auf Wertpapierkurse im Rahmen von Rating-Änderungen

\section{Ereignisstudien zur Messung der Reaktionen von Wertpapierrenditen auf}

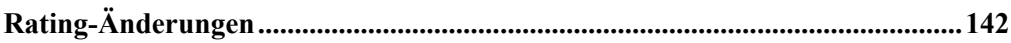

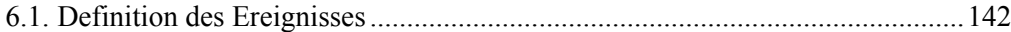

6.2. Berechnung der abnormalen Rendite ......................................................... 145

6.3. Wertpapierspezifische Anpassungen bei der Berechnung abnormaler

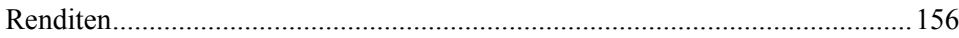

6.4. Verfahren zur statistischen Interpretation der Ergebnisse................................ 164

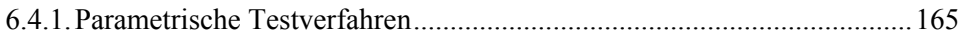

6.4.2. Nichtparametrische Testverfahren ........................................................ 167

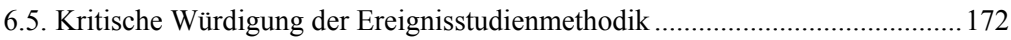

6.6. Überblick über die Konzeption bisheriger Ereignisstudien.............................. 175

6.6.1. Konzeptionsdetails bisheriger Ereignisstudien ...................................... 175

6.6.2. Konzeptionelle Schwachstellen bisheriger Ereignisstudien .................... 185

7. Empirische Analyse des Ankündigungseffektes von Credit Ratings....................190

7.1. Beschreibung verwendeter Daten................................................................... 190

7.1.1. Beschreibung der erhobenen Rating-Änderungen ................................. 190

7.1.2. Beschreibung der erhobenen Kursdaten ...............................................202

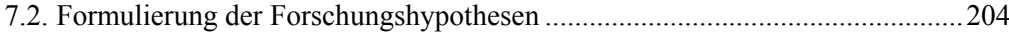

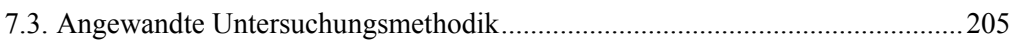




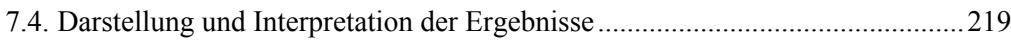

7.4.1. Gesamtergebnisse je Wertpapierkategorie...........................................219

7.4.2. Ergebnisse nach Subsamples: Branchenherkunft des Emittenten............225

7.4.3. Ergebnisse nach Subsamples: konjunktureller Einfluss..........................232

7.4.4. Analyse des Einflusses unabhängiger Variablen ..................................237

7.4.4.1. Reaktion der abnormalen Renditen von Aktien ........................238

7.4.4.2. Reaktion der abnormalen Renditen von Unternehmens-

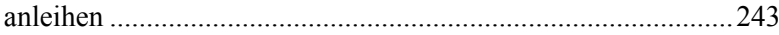

7.4.4.3. Reaktion der abnormalen Renditen von Wandelanleihen ..........247

7.4.5. Analyse der Reaktionsstärke .............................................................250

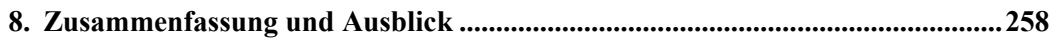

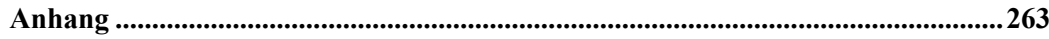

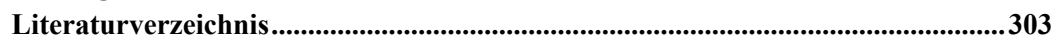




\section{Abbildungsverzeichnis}

Abb. 1: Kursentwicklung auf halbstreng informationseffizienten Wertpapiermärkten .. 18

Abb. 2: Definitionsvergleich des Rating-Begriffs aus Sicht der Rating-Unternehmen ..25

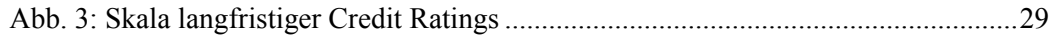

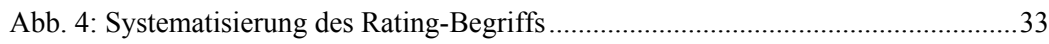

Abb. 5: Exemplarischer Ablauf des Erstellungsprozesses eines Credit Ratings.............39

Abb. 6.1: Geografische Einteilung angekündigter Rating-Änderungen der drei führenden Rating-Unternehmen p.a. .....................................................................46

Abb. 6.2: Europäische Marktanteile der drei führenden Rating-Unternehmen gemessen an der Anzahl angekündigter Rating-Änderungen p.a................................46

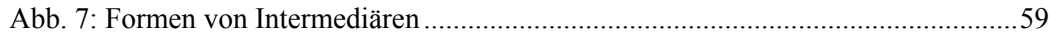

Abb. 8: Informationsasymmetrien und opportunistisches Verhalten.............................65

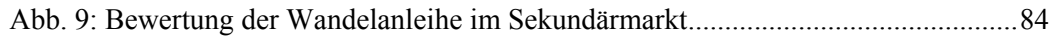

Abb. 10: Bisherige Studien mit Fokus europäische Wertpapiermärkte ..........................89

Abb. 11: Bisherige Studien mit Fokus auf den Werttransfer zwischen Aktionär und

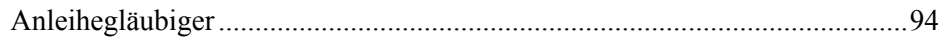

Abb. 12: Bisherige Studien mit Fokus auf die Branchenherkunft des Emittenten ........101

Abb. 13: Anzahl angekündigter Rating-Änderungen im Zeitraum 2000 bis 2010 .......108

Abb. 14: Bisherige Studien mit Fokus auf den Konjunkturzyklus ...............................109

Abb. 15: Handelsvolumen und Transaktionsanzahl des europäischen Unternehmensanleihe- und Aktienmarktes im Zeitraum 2007 bis 2010 ............................ 113

Abb. 16: Handelsvolumen und Transaktionsanzahl des europäischen Unternehmensanleihe- und Aktienmarktes im Zeitraum 2003 bis 2006 ............................114

Abb. 17: Pecking Order ausgewählter Formen verbriefter Finanzierung .....................129

Abb. 18.1: Rating-Kriterien hybrider Finanzinstrumente nach Fitch ..........................133

Abb. 18.2: Rating-Kriterien hybrider Finanzinstrumente nach Moody's .....................134

Abb. 18.3: Rating-Kriterien hybrider Finanzinstrumente nach Standard \& Poor's...... 134

Abb. 19: Eigenkapitalcharakter ausgewählter Finanzinstrumente ...............................136

Abb. 20: Eigenkapitalcharakter ausgewählter Finanzinstrumente …...........................138

Abb. 21: Systematisierung bisheriger Indexarten zur Berechnung der Marktrendite... 150

Abb. 22: Darstellung der zeitlichen Konzeption einer Ereignisstudie..... .155 
Abb. 23: Einfache OLS-Regression mit Ausreißern und Leverage Points

Abb. 24: Darstellung der zeitlichen Konzeption des GRANK-Tests.....

Abb. 25: Ausgewählte Hypothesen bisheriger Studien.

Abb. 26: Anteil statistischer Signifikanztests in bisherigen Studien 186

Abb. 27: Darstellung der enthaltenen double counting events 191

Abb. 28: Systematisierung des verwendeten Rating-Begriffs 193

Abb. 29: Anzahl untersuchter Rating-Änderungen. 195

Abb. 30: Zeitliche Verteilung der untersuchten Rating-Änderungen p.a. 198

Abb. 31: Beschreibung der Split Ratings von Stichprobenunternehmen. 199

Abb. 32: Verteilung der untersuchten Rating-Änderungen in der Migrationsmatrix ...201

Abb. 33: Länderübergreifende Indizes pro Wertpapierkategorie zur Berechnung von $\mathrm{R}_{\mathrm{M}, \mathrm{t}}$ 209

Abb. 34: Vergleich der Regressionsgüte zwischen nationalen und länderübergreifenden Indizes 210

Abb. 35: Vergleich der Regressionsgüte zwischen OLS- und LTS-Regression .211

Abb. 36.1: OLS- und LTS-Regressionsanalyse pro Wertpapierkategorie: Stichprobenunternehmen - ADECCO S.A.

Abb. 36.2: OLS- und LTS-Regressionsanalyse pro Wertpapierkategoire: Stichprobenunternehmen - Compagnie Generale des Etablissements Michelin SCA...215

Abb. 37: p-Werte des Shapiro-Wilk-Tests pro Wertpapierkategorie..... .218

Abb. 38: CAR-Verläufe pro Wertpapierkategorie und Art der Rating-Änderung ........220

Abb. 39: Standardisierte kumulierte abnormale Renditen (SCARs) und standardisierte abnormale Renditen (SARs) pro Wertpapierkategorie im Rahmen von Upgrades und Downgrades. 221

Abb. 40: SCARs und SARs in Abhängigkeit der Branchenherkunft des Emittenten...227

Abb. 41: SCARs und SARs in Abhängigkeit des Konjunkturzyklus 233

Abb. 42: Vergleich der berechneten CARs mit den Resultaten von Jaballah (2012) ...235

Abb. 43: Regressionskoeffizienten unabhängiger Variablen in Bezug auf abnormale Renditen von Aktien im Rahmen von Rating-Änderungen

Abb. 44: Regressionskoeffizienten unabhängiger Variablen in Bezug auf abnormale Renditen von Unternehmensanleihen im Rahmen von Rating-Änderungen .244

Abb. 45: Regressionskoeffizienten unabhängiger Variablen in Bezug auf abnormale Renditen von Wandelanleihen im Rahmen von Rating-Änderungen 248 
Abb. 46: SCAR-Vergleich zwischen zwei Wertpapierkategorien .253

Abb. 47: Monatliche Geld-Brief-Spanne der untersuchten Wertpapiere .255

Abb. 48: Zusammenfassende Resultate getesteter Hypothesen .260

Abb. A1: Kumulierte abnormale Renditen (CARs) und abnormale Renditen (ARs) pro

Wertpapierkategorie im Rahmen von Upgrades und Downgrades..... .263

Abb. A2: CARs und ARs in Abhängigkeit der Branchenherkunft des Emittenten ......264

Abb. A3: CARs und ARs in Abhängigkeit des Konjunkturzyklus .265

Abb. A4: Europäischer Primärmarkt für Wandelanleihen im Zeitraum 2000 bis 2010266

Abb. A5: Wachstumsrate europäischer Wertpapierindizes p.a. von 2003 bis 2006 .....266

Abb. A6.1: Verwendete nationale Aktienindizes zur Berechnung von $\mathrm{R}_{\mathrm{M}, \mathrm{t}}$ 267

Abb. A6.2: Verwendete nationale Wandelanleiheindizes zur Berechnung von $\mathrm{R}_{\mathrm{M}, \mathrm{t}} \ldots .267$

Abb. A6.3: Verwendete nationale Unternehmensanleiheindizes zur Berechnung von $\mathrm{R}_{\mathrm{M}, \mathrm{t}}$ 268

Abb. A7: Erzielte Rendite europäischer Hedgefonds im Rahmen der Convertible Arbitrage Strategie .268

Abb. A8: Untersuchte Hypothesen in bisherigen Studien von 1974 bis 2013 .269

Abb. A9: Allgemeine Konzeptionsdetails bisheriger Studien im Zeitraum 1974 bis 2013 .275

Abb. A10.1: Spezifische Konzeptionsdetails bisheriger Studien im Zeitraum 1974 bis 2013 (Teil 1) 281

Abb. A10.2: Spezifische Konzeptionsdetails bisheriger Studien im Zeitraum 1974 bis 2013 (Teil 2) .289

Abb. A11: Auflistung der analysierten Stichprobenunternehmen 300 


\section{Abkürzungsverzeichnis}
Abb.
Abbildung
AEX
Amsterdam Exchanges Index
AISBL
Association Internationale Sans But Lucratif
ASE/ATHEX
Athens Stock Exchange
ASX
Australian Securities Exchange
ATX
Austrian Traded Index
BEL
Belgian Index
Bd.
Band
BOVESPA Bolsa de Valores de São Paulo
bzw. beziehungsweise
ca.
circa
CAC
Cotation Assistée en Continu
CDAX
Composite Deutscher Aktienindex
CDS
Credit Default Swap
Corp. Corporation
CRSP Center for Research in Security Prices
DAX Deutscher Aktienindex
DCR Duff \& Phelps Credit Rating Co.
DJTM Dow Jones Total Market
EBIT Earnings before Interest and Tax
EBITDA Earnings before Interest, Tax, Depreciation and Amortization
ed. edition
EDV Elektronische Datenverarbeitung
EMEA Europe, Middle East, Africa
etc. et cetera
EU Europäische Union
EUR Euro
f. folgende (Seite) 


\begin{tabular}{|c|c|}
\hline FESE & Federation of European Securities Exchanges \\
\hline ff. & fortfolgende (Seiten) \\
\hline FN & Fußnote \\
\hline FTA & Financial Times Actuaries Share Index \\
\hline FTSE & Financial Times Stock Exchange \\
\hline GE & Geldeinheiten \\
\hline ggf. & gegebenenfalls \\
\hline Hrsg. & Herausgeber \\
\hline i.e.S. & im engeren Sinne \\
\hline i.w.S. & im weiteren Sinne \\
\hline IAS & International Accounting Standard \\
\hline IBEX & Iberia Index \\
\hline IFRS & International Financial Reporting Standard \\
\hline Inc. & Incorporation \\
\hline insbes. & insbesondere \\
\hline IPC & Índice de Precios y Cotizaciones \\
\hline IPSA & Indice de Precios Selectivo de Acciones \\
\hline ISEQUIT & Ireland Stock Exchange Overall Index \\
\hline ISIN & International Securities Identification Number \\
\hline Kap. & Kapitel \\
\hline KOSPI & Korea Composite Stock Price Index \\
\hline Ifd. $\mathrm{Nr}$. & laufende Nummer \\
\hline LLC & Limited Liability Company \\
\hline LLP & Limited Liability Partnership \\
\hline Ltd. & Limited \\
\hline LUXX & Luxembourg Stock Exchange \\
\hline MERVAL & Mercado de Valores de Buenos Aires \\
\hline MIB & Milano Indice Borsa \\
\hline Mrd. & Milliarden \\
\hline MSCI & Morgan Stanley Capital Index \\
\hline
\end{tabular}




\begin{tabular}{|c|c|}
\hline N.V. & Naamloze Vennootschap \\
\hline NASDAQ & $\begin{array}{l}\text { National Association of Securities Dealers for Automate } \\
\text { Quotation }\end{array}$ \\
\hline NYSE AMEX & New York and American Stock Exchange \\
\hline NZSE & New Zealand Stock Exchange \\
\hline o.g. & oben genannte(n) \\
\hline OECD & Organisation for Economic Co-operation and Development \\
\hline OMX & Optionsmäklarna Stock Exchange \\
\hline OMXH & Optionsmäklarna Stock Exchange Helsinki \\
\hline p.a. & per annum \\
\hline plc & Public Limited Company \\
\hline PSI & Portuguese Stock Index \\
\hline RoA & Return on (Total) Assets \\
\hline $\mathbf{R Z}$ & Randziffer \\
\hline S\&P's & Standard \& Poor's \\
\hline S. & Seite(n) \\
\hline S.A. & Société Anonyme, Sociedade Anónima \\
\hline S.C.A. & Société en Commandite par Actions \\
\hline SE & Societas Europaea \\
\hline S.p.A. & Società per Azioni \\
\hline SBI & Swiss Bond Index \\
\hline SCA & Société en commandite par actions \\
\hline SIC & Standard Industrial Classification \\
\hline TOPIX & Tokyo Stock Price Index \\
\hline ToR & Total Return (Gesamtrendite) \\
\hline TRACE & Trade Reporting and Compliance Engine \\
\hline Tsd. & Tausend \\
\hline u.a. & unter anderem \\
\hline u.ä. & und ähnliches \\
\hline U.S./US & United States \\
\hline
\end{tabular}


u.U. unter Umständen

USD U.S. Dollar

VC Venture Capital

Verf. Verfasser

vs. versus

z.B. zum Beispiel 